\title{
RIEMANN-ROCH THEOREMS FOR HIGHER ALGEBRAIC $K$-THEORY
}

BY HENRI GILLET ${ }^{1}$

In [1] and [2] Baum, Fulton and MacPherson, generalizing the celebrated Grothendieck-Riemann-Roch theorem, proved that given a category $V$ of quasi-projective schemes there is a natural transformation called the Todd class of functors (covariant for proper morphisms) between $K_{0}^{\prime}$, the homology algebraic $K$-theory of coherent sheaves and any of the standard homology theories. Here we announce generalizations of the results of [1] and [2] to Quillen's higher algebraic $K$-theory [8] which may help to illuminate the relationship between algebraic $K$-theory and more ordinary cohomology theories.

The statements of our theorems depend on defining global analogues of Quillen's construction of Chern classes for the $K$-theory of a ring [3], [9]. We can use any of the standard cohomology theories defined on $V$, such as étale or crystalline cohomology or even the Chow ring. All of these theories can be realized for each $X \in V$ as the hypercohomology of a graded complex or procomplex $\Gamma_{j}^{*}, j \in Z$, of sheaves on the Zariski site of $X$. All of these theories have Chern classes for representations of sheaves of groups and there exist universal classes

$$
C_{i} \in \mathbf{H}^{d i}\left(X, G L\left(O_{X}\right), \Gamma_{i}^{*}\right) \quad(d=1 \text { or } 2) .
$$

Using Brown's generalized cohomology "with supports" of simplicial sheaves [6], and the functor $Z_{\infty}$ of [5] instead of the " + " construction one can mimic in the category of simplicial sheaves the methods of [3] and [9] to obtain Chern classes for all $p>0$

$$
C_{i, p}^{Y} ; K_{p}^{Y}(X)=K_{p}(X, X-Y) \rightarrow \mathrm{H}_{Y}^{d i-p}\left(X, \Gamma_{i}^{*}\right)
$$

whose domains are the relative $K$-groups, defined so as to force a Quillen-style localization sequence. One can show that these classes coincide for $p=0$ with those of Iversen [7]. For $p>0$ they are group homomorphisms and are compatible with products in the way described by Bloch [3], hence one can define a Chern character with supports, which is a ring homomorphism

$$
\text { ch. } Y: \bigoplus_{p \geqslant 0} K_{p}(X, X-Y) \rightarrow \bigoplus_{i, p \geqslant 0} H_{Y}^{d i-p}\left(X, \Gamma_{i}^{*}\right) \otimes \mathbf{Q}
$$

Received by the editors January 29, 1980.

1980 Mathematics Subject Classification. Primary 14F12, 14C35; Secondary 18F25. 1 Partially supported by an NSF grant. 
THEOREM 1. For $Y \in V$ define the $\Gamma$-homology groups $H_{i}\left(Y, \Gamma_{j}\right)$ to be $\mathbf{H}_{Y}^{d n-i}\left(X, \Gamma_{n-j}^{*}\right)$ where $Y \subset X$ and $X$ is smooth of dimension $n$. Then there is a natural transformation of covariant functors on the category of proper morphisms in $V$, with domain the $K^{\prime}$-theory of coherent sheaves [8] ,

$$
\tau_{*}=\bigoplus_{p \geqslant 0} \tau_{p}: \bigoplus_{p \geqslant 0} K_{p}^{\prime}(Y) \rightarrow \bigoplus_{p, i \geqslant 0} H_{d i+p}\left(Y, \Gamma_{i}\right) \otimes \mathbf{Q} .
$$

$\tau_{*}$ satisfies the following conditions:

(i) For all $X \in V$ and $\alpha \in K_{p}(X), \beta \in K_{q}^{\prime}(X)$,

$$
\tau_{p+q}(\alpha \cap \beta)=\operatorname{ch}_{p}(\alpha) \cap \tau_{q}(\beta) .
$$

(ii) If $X, Y \in V$ and $\alpha \in K_{p}^{\prime}(X), \beta \in K_{q}^{\prime}(Y)$ then $\tau(\alpha \otimes \beta)=\tau(\alpha) \otimes \tau(\beta)$

$$
\text { ( } \otimes=\text { external product in } K^{\prime} \text {-theory or } \Gamma \text {-homology). }
$$

(iii) If $j: U \rightarrow X$ is an open immersion in $V$ and $\alpha \in K_{p}^{\prime}(X)$ then $\tau_{p}(j * \alpha)=j \tau_{p}(\alpha)$

(iv) If $X \in o b(V)$ is smooth then the structure sheaf $O_{X}$ defines an element $\left[0_{X}\right]$ of $K_{0}^{\prime}(X)$ and $\tau_{0}\left[0_{X}\right]=\operatorname{Td}(X) \cap \eta_{X}$, where $\operatorname{Td}(X)$ is the classical Todd class and $\eta_{X} \in H_{d n}\left(X, \Gamma_{n}\right)$ is the homology cycle class of $X(n=$ dimension of $X)$.

The construction of $\tau_{*}$ and the proof of Theorem 1 are adapted from the methods of [1]. However the extension to higher $K$-theory does involve more detailed "functorial" constructions than are necessary for $K_{0}$. Similar methods to those of Theorem 1 and the paper [2] can be used to prove

THEOREM 2. On the category of quasi-projective algebraic varieties over $\mathbf{C}$ there is a natural transformation

$$
\tau_{*}=\bigoplus_{p \geq 0} \tau_{p}: \underset{p \geq 0}{\bigoplus_{p}} K_{p}^{\prime} \rightarrow \bigoplus_{p \geq 0} K U_{p}^{L C}
$$

covariant for proper morphisms, where $K U_{p}^{L C}$ is the ' $L-C$ ' or 'locally compact' homology topological $K$-theory associated to the spectrum $B U$. There is also a natural transformation

$$
\tau^{*}: K^{*} \rightarrow K U^{*}
$$

These maps $\tau_{*}, \tau^{*}$ satisfy the "module", "product" and presheaf properties analogous to (i), (ii) and (iii) of Theorem 1.

If $X$ is a Noetherian scheme, there is a filtration

$$
N^{*} X=\left\{X^{(0)}=X \supset X^{(1)} \supset \cdots \supset X^{(n)} \supset \cdots\right\}
$$

of its underlying topological space, called the coniveau filtration, defined by $X^{(k)}=\{x \in X \mid\{\bar{x}\}$ has codimension $\geqslant k$ in $X\}$. 
For any simplicial sheaf $F$. on $X$, there is a natural exact couple associated to $N^{*} X$ and hence a spectral sequence [8], [3]:

$$
E_{1}^{p q}\left(F_{0}\right)=\bigoplus_{x \in X^{(p)}-X^{(p+1)}} H_{x}^{p+q}\left(X, F_{.}\right) \Rightarrow H^{p+q}(X, F .) .
$$

Given a cohomology theory $\bigoplus_{i \geq 0} \Gamma_{i}^{*}$, for each $X \in V$ one obtains maps of coniveau spectral sequences

$$
E_{r}^{p q}\left(C_{i}\right): E_{r}^{p q}\left(\mathrm{Z}_{\infty} B . G L\left(O_{X}\right)\right) \rightarrow E_{r}^{p q}\left(K\left(d i, \Gamma_{i}^{*}\right)\right)
$$

which on $E_{1}$ terms may be written

$\bigoplus C_{i,-p-q}^{x}$ :

$$
\begin{aligned}
& \underset{x \in X^{(p)}-X_{-X}^{(p+1)}}{\Pi_{-p-q}^{x}(X) \longrightarrow} \underset{x \in X^{(p)}}{\bigoplus_{-X}^{(p+1)}} \mathbf{H}_{x}^{d i+p+q}\left(X, \Gamma_{i}^{*}\right) \\
& \downarrow \int \quad \downarrow \int \\
& \underset{x \in X^{(p)}}{\bigoplus_{-X}^{(p+1)}} K_{-p-q}(\mathrm{k}(x)) \stackrel{\gamma_{i}^{x}}{\rightarrow} \underset{x \in X^{(p)}}{\bigoplus_{-X}^{(p+1)}} \mathbf{H}^{d(i-p)+p+q}\left(x, \Gamma_{i-p}^{*}\right)
\end{aligned}
$$

where the vertical isomorphisms come from duality and Quillen's localization theorem [8].

THEOREM 3. The map $\gamma_{i}^{x}$ in the diagram above is equal to $(-1)^{p}(i-1) ! /(i-p-1) ! C_{i-p,-p-q}$.

This is a consequence of the following Riemann-Roch theorem without denominators and with supports.

THEOREM 4. Let $j: Y \rightarrow X$ be a closed immersion of smooth schemes in $V$. Then we have isomorphisms

$$
\begin{aligned}
& j_{*}: K_{q}(Y) \stackrel{\sim}{\longrightarrow} K_{q}(X, X-Y), \\
& j_{!}: \mathbf{H}^{d(i-p)-q}\left(Y, \Gamma_{i-p}^{*}\right) \rightarrow \mathbf{H}_{Y}^{d i-q}\left(X, \Gamma_{i}^{*}\right)
\end{aligned}
$$

where $p=\operatorname{codim}_{X}(Y)$. For each $k \geqslant 0$ there exist polynomials $P_{k}\left(T_{0}, \ldots, T_{k-p}\right.$; $\left.U_{1}, \ldots, U_{k-p}\right)$ with integer coefficients such that if $\alpha \in K_{q}(Y)$ and $q \leqslant 2 k$ then

$$
C_{k}^{Y}\left(j_{*} \alpha\right)=j_{!}\left(P_{k}\left(r k, C_{1}, \ldots, C_{k-p} ; C_{1}\left(N_{X / Y}\right), \ldots, C_{k-p}\left(N_{X / Y}\right)\right)(\alpha)\right) .
$$

Here $r k, C_{1}, \ldots, C_{k-p}$ are the universal Chern classes in $\mathrm{H}^{*}\left(Y, G L\left(O_{Y}\right), \Gamma_{*}^{*}\right)$ and the $C_{i}\left(N_{X / Y}\right)$ are the Chern classes of the normal bundle of $Y$. The polynomial $P_{k}$ in these classes defines a class in $\mathbf{H}^{d k}\left(Y, G L\left(O_{Y}\right), \Gamma_{k}^{*}\right)$ which we apply to $\alpha$.

Theorem 4 is proved by using the "deformation to the normal bundle" construction of [1] to reduce to the case of the zero section $Y \subset N_{X / Y}$ followed by explicit computations. The same methods may be used to prove Theorems 3 
and 4 in the case where $X$ is any connected regular one-dimensional scheme and $\Gamma^{*}$ is étale cohomology, in which case $j_{1}$ is defined by Grothendieck's absolute purity theorem (SGA5, I §5.1). In the case $X=\operatorname{Spec}\left(O_{S}\right), O_{S}$ the ring of $S$-integers in a global field, this extends more ad-hoc results of Soule in [9].

\section{REFERENCES}

1. P. Baum, W. Fulton and R. MacPherson, Riemann-Roch for singular varieties, Inst. Hautes Études Sci. Publ. Math. 45 (1975), 101-146.

2. - Riemann-Roch and topological K-theory, Acta. Math. (to appear).

3. S. Bloch, Algebraic $K$-theory and crystalline cohomology, Inst. Hautes Études Sci. Publ. Math. 47 (1977), 187-268.

4. S. Bloch and A. Ogus, Gesten's conjecture and the homology of schemes, Ann. Sci. Ecole Norm. Sup. 7 (1974), $181-202$.

5. A. K. Bousfield and D. M. Kan, Homotopy limits, completions and localizations, Lecture Notes in Math., vol. 304, Springer-Verlag, Berlin and New York, 1972.

6. K. Brown, Abstract homotopy theory and generalized sheaf cohomology, Trans. Amer. Math. Soc. 186 (1973), 419-458.

7. B. Iversen, Local Chern classes, Ann. Sci. École Norm. Sup 9 (1976), 155-169.

8. D. Quillen, Higher algebraic K-theory. I, Lecture Notes in Math., vol. 341, SpringerVerlag, Berlin and New York, 1972.

9. C. Soulé, Thesis, University of Paris, 1979.

DEPARTMENT OF MATHEMATICS, PRINCETON UNIVERSITY, FINE HALL, BOX 37, PRINCETON, NEW JERSEY 08544 